\title{
Fortschritte der Allergologie - Allergologie im Aufwind
}

D ie Überschrift nennt die Tagungsthemen zweier wichtiger Allergiekongresse in den nächsten Wochen, des 18. Fortbildungskongresses in Davos mit Dreiländertreffen der Deutschen, Schweizerischen und Österreichischen Gesellschaften für Allergologie und des 27. ÄDA-Kongresses in Dresden. Abstracts (S. 364) bzw. Programm (S. 397) finden Sie in diesem Heft. Außerdem eine Nachbetrachtung zur allergica in Frankfurt/Main und die Anmeldungen zum 15. Mainzer Allergie-Workshop 2003 - diese Ausgabe des

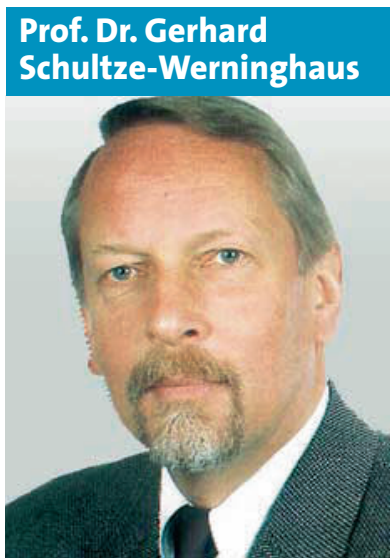

Abteilung für Pneumologie, Allergologie und Schlafmedizin, BG Kliniken Bergmannsheil Universitätsklinik, Bochum

\section{Prof. Dr. Bernhard}

Przybilla



Klinik und Poliklinik für Dermatologie und Allergologie, Klinikum der Ludwig-MaximiliansUniversität München AlLERGO-Journals belegt die

Fülle allergologischer Fortbil-

dungsmöglichkeiten und es ist zu hoffen, dass möglichst viele unserer Leser die Gelegenheiten nutzen können. Den Kollegen in Dresden gilt in diesen Tagen der Flut unser besonderes Mitgefühl im Vorfeld des ÄDA-Kongresses.

Was ist neu? Was lässt sich schon in der Praxis umsetzen? Diesen Fragen sehen sich Veranstalter ausgesetzt - zu Recht, angesichts des finanziellen sowie zeitlichen Aufwandes einer $\mathrm{Ta}$ gungsteilnahme.

Die Durchsicht

der Tagungsinhalte in diesem Heft wie auch der übrigen Beiträge macht deutlich, dass in der Grundlagenforschung sowie in Klinik und Praxis in der Tat eine Reihe zentraler Aspekte in neuem Licht dasteht, so dass die Beschäftigung mit der Materie neue Horizonte erkennen lässt.

Um zwei Aspekte herauszugreifen: Besonders faszinierend in der immunologischen Grundlagenforschung ist die Fülle neuer Daten, die zu belegen scheinen, dass die Interaktion zwischen maternalem und fetalem Immunsystem für das gesamte Leben wichtige Weichen stellt - nicht absehbar, welche Möglichkeiten sich durch interventionelle Ansätze ergeben könnten, besonders in der Allergieprävention. Endlich scheinen Antworten auf die Fragen nach der Ursache und der Prävalenzzunahme allergischer Krankheiten möglich.

In Klinik und Praxis ist die Spezifische Immuntherapie (SIT) ein wichtiger Anlass, um eine sorgfältige Allergiediagnostik durchzuführen, für die Berechtigung der Allergologie als eigene Fachrichtung ist die SIT eine wichtige Begründung. Daher ist der neue optimistische



Prof. Dr. G. Schultze-Werninghaus flügeln wird.

Vor 100 Jahren veröffentlichten Paul Portier und Charles Richet ihre bahnbrechenden Daten über Versuche zur (von ihnen so benannten) Anaphylaxie, die sie ein Jahr zuvor auf einer vom Fürsten Albert I. von Monaco finanzierten Studienfahrt von Monaco bis zu den Kapverdischen Inseln auf der fürstlichen Jacht „Princesse Alice II“ durchgeführt hatten. Richet erhielt hierfür 1913 den Nobelpreis. Dies zeigt nicht nur, dass projektbezogene Forschungsförderung zu außerordentlichem Erkenntnisgewinn führen kann, sondern auch, dass es trotz der 100 Jahre einer wissenschaftlichen Allergologie noch eine Fülle unbeantworteter Fragen gibt. Es bleibt spannend!

Mit den besten Grüßen

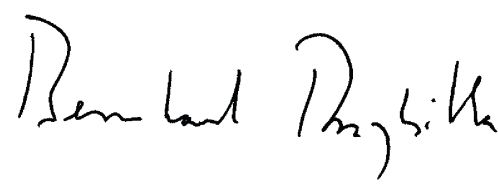

Prof. Dr. B. Przybilla 